\title{
Branched-chain amino acids does not improve muscle recovery from resistance exercise in untrained young adults
}

José Maria Estoche ${ }^{1}$, Jeferson Lucas Jacinto ${ }^{1}$, Mirela Casonato Roveratti ${ }^{1}$, Juliano Moro Gabardo $^{1}$, Cosme Franklim Buzzachera ${ }^{1}$, Erick Prado de Oliveira ${ }^{3}$, Alex Silva Ribeiro ${ }^{1}$, Rubens Alexandre da Silva ${ }^{1,2}$, Andreo Fernando Aguiar ${ }^{1}$

${ }^{1}$ Center of Research in Health Sciences, North University of Paraná (UNOPAR), Londrina, Paraná, Brazil.

${ }^{2}$ Département des Sciences de la Santé, Programme de Physiothérapie de L’université McGill Offert em Extension à l'université du Québec à Chicoutimi (UQAC) et Laboratoire de recherche BioNR, 555 boul. De L’université, ville du Saguenay, Québec, QC G7H 5B8, Canada.

3 School of Medicine, Federal University of Uberlandia, Uberlandia, Minas Gerais, Brazil.

Corresponding author: Andreo Fernando Aguiar ( $\bowtie)$

Center of Research in Health Sciences, North University of Paraná (UNOPAR), Avenue Paris, 675, Jardim Piza, CEP: 86041-120, Londrina, PR, Brazil. Tel: +55 4399523813, Fax: +55 4333717725, Email: afaguiarunesp@gmail.com 


\section{ABSTRACT}

The purpose of this study was to investigate the effects of BCAA supplementation on muscle recovery from resistance exercise (RE) in untrained young adults. Twenty-four young adults ( $24.0 \pm 4.3$ years old) were assigned to 1 of 2 groups ( $n=12$ per group): a placebo-supplement group or an BCAA-supplement group. The groups were supplemented for a period of 5 days. On day 1 and 3, both groups underwent a RE session involving two lower body exercises (hack squat and leg press) and then were evaluated for muscle recovery on the 3 subsequent moments after the RE session (30 min. [day 3], $24 \mathrm{~h}$ [day 4], and $48 \mathrm{~h}$ [day 5]). The following indicators of muscle recovery were assessed: number of repetitions, rating of perceived exertion in the last RE session, muscle soreness and countermovement jump (CMJ) during recovery period (30 min., $24 \mathrm{~h}$, and $48 \mathrm{~h}$ after RE session). Number of repetitions remained unchanged over time (time, $P>0.05$ ), while the rating of perceived exertion increased (time, $P<$ 0.05 ) over 3 sets, with no difference between groups (group x time, $P>0.05$ ). Muscle soreness increased (time, $P<0.05$ ) and jumping weight decreased (time, $P<0.05$ ) at 30 min. postexercise and then progressively returned to baseline at 24 and $48 \mathrm{~h}$ postexercise, with no difference between groups (group x time, $P>0.05$ ). The results indicate that BCAA supplementation does not improve muscle recovery from RE in untrained young adults.

keywords: supplementation; essential amino acids; muscle function; strength exercise; muscle soreness; muscle power. 


\section{INTRODUCTION}

Protein and amino acids supplements are frequently consumed by athletes and recreationally-active adults to improve physical performance and muscle recovery. In particular, the branched-chain amino acids (BCAA) family, leucine, isoleucine and valine, has received special attention in the last 35 years because of their supposed effects on muscle protein synthesis (Wolfe et al. 2017). BCAA are three of the nine essential amino acids (EAAs) that comprise muscle (14-18\% of the total EAAs) and body (35-40\% of the total EAAs) proteins (Riazi et al. 2003; Layman and Baum, 2004; Shimomura et al. 2006), and along with the eleven non-essential amino acids (NEAAs) are responsible for the synthesis of new muscle protein (Wolfe et al. 2017).

BCAA, especially leucine, has been shown to enhance muscle protein synthesis and reduce breakdown after exercise by stimulating the phosphorylation of anabolic factors (i.e., mammalian target of rapamycin [mTOR] and 70-kDa S6 protein kinase [p70 ${ }^{\text {s6k }}$ ] (Karlsson et al., 2004; Blomstrand et al. 2006; Jackman et al. 2017) and reducing gene expression of catabolic factors, such as muscle RING-finger 1 (MuRF-1) and muscle atrophy F-box (MAFbx) (Borgenvik et al. 2012). Despite this supposed anabolic propriety, the effects of BCAA supplementation on markers of muscle recovery remains controversial. While several studies have shown beneficial effects on muscle soreness (Jackman et al. 2010; Shimomura et al. 2006; Shimomura et al. 2010; Howatson et al. 2012; Kim et al. 2013; Greer et al. 2007), muscle strength (Shimomura et al. 2010; Howatson et al. 2012; Greer et al. 2007) and creatine kinase (CK) activity (Howatson et al. 2012; Sharp and Pearson, 2010; Nosaka et al. 2006), others have not (Knechtle et al. 2012; Ra et al. 2013; Areces et al. 2014; Pasiakos et al. 2014; Fouré et al. 2016). 
The explanation for these conflicting results remains unknown but may be related to differences in the study designs (e.g., dosage, period, and supplementation timing), subjects' training status, and the large interindividual variability in levels of indirect markers of muscle damage (e.g., CK). Another recurring limitation in several studies is the lack of dietary monitoring, mainly in relation to dietary protein intake, which may markedly confound the interpretation of the results. Moreover, it is important to mention that most of the studies involving BCAA supplementation on recovery markers have reported a mix of muscle damage (i.e., from low-to-high) and exercise types (i.e., eccentric exercise, drop jumps, strength exercises, running, and cycling ergometer) (for a review see Fouré and Bendahan, 2017), making it difficult to establish a consensus on the ergogenic effects of BCAA during recovery process. Therefore, well-controlled studies are warranted to confirm whether supplementation of BCAA could alleviate the alterations of muscle soreness and function during time course of recovery from exercise, mainly in response to a conventional resistance exercise (RE) session.

The purpose of this study was to investigate the effects of BCAA supplementation on muscle recovery from RE in untrained young adults. Considering previous positive findings on muscle anabolism following exercise (Karlsson et al., 2004; Blomstrand et al. 2006; Jackman et al. 2017), we hypothesized that BCAA supplementation would ameliorate the negative symptoms associated with highintensity RE stimulus, including muscle soreness and loss of muscle function (i.e., number of maximum repetitions, and jumping power output), when compared with the placebo. 


\section{METHODS}

\section{Participants}

Twenty-four healthy individuals were recruited from a university population, and all completed the study. Descriptive characteristics of the participants are presented in Table 1. An a priori power analysis was conducted ( $G^{*}$ Power version 3.0.1) for an $F$ test (repeated measures, within-between interaction factors for 4 time points). On the basis of a statistical power $(1-\beta)$ of 0.90 , a moderate effect size (0.5), and an overall level of significance of 0.05 , at least 10 participants were required in each group. Participants were included if they (i) had not ingested any ergogenic supplement or anabolic steroid during the 6 months prior to the start of the study, (ii) were not taking any medication that could affect muscle recovery or the ability to train intensely, (iii) had not participated in a exercise training program for more than 2 days per week during the 6 months prior to the beginning of the study, (iv) were able to provide a detailed description of their lifestyle and daily food intake, or $(v)$ did not have medical approval to perform physical exercise. All participants were informed of the procedures, risks, and benefits of the investigation and signed an informed consent document approved by the Institutional Review Board of the University (protocol \#1.748.002). All procedures were carried out in accordance with the ethical standards as laid down in the 1964 Declaration of Helsinki and its later amendments or comparable ethical standards.

\section{Experimental design}

A double-blind, placebo-controlled, parallel-groups design with repeated measures was performed to examine the effects of BCAA supplementation on the time course of muscle recovery from RE in young adults (Fig. 1). Initially, all participants completed 3 sessions of tests of 10-repetition maximum (10RM) for leg press and hack squat exercises, following 2 familiarization sessions with countermovement jump (CMJ) test, 
OMNI-RES scale (rating of perceived exertion), visual analogic scale (VAS, muscle soreness), and exercises (leg press and hack squat) in order to minimize any potential learning effect. Thereafter, the participants were matched according to sex and 10RM strength and then randomly assigned to a BCAA or placebo group ( $N=12$ per group; 9 men and 3 women in each group), who were supplemented for a period of 5 days. On day 1 and 3, both groups underwent a session of RE and then evaluated for muscle recovery on the 3 subsequent moments (30 min. [day 3], 24 h [day 4], and 48 h [day 5]) after the RE session. The following indicators of muscle recovery were assessed: number of repetitions and rating of perceived exertion in the last RE session (day 3), and muscle soreness and countermovement jump (CMJ) during recovery period (30 min., $24 \mathrm{~h}$, and $48 \mathrm{~h}$ after last RE session). Moreover, all participants completed a 3-day dietary intake record between RE sessions (days 1-3) to monitor any influence of diet.

\section{** Figure 1 about here **}

\section{Determination of 10RM load}

Before randomization, all participants completed 3 sessions of leg press and hack squat 10RM tests, using standard protocols previously documented elsewhere (da Silva et al. 2017; Andrade et al. 2018). The test was preceded by a warm-up exercise ( 15 repetitions) at approximately 50\% of the load to be used in the first attempt of the test. After 2 min of rest, the attempts were performed with a progressively increasing load (5\%-10\%) for each attempt and were separated by 4 - to 5-min rest intervals to allow adequate recovery. Only 3 attempts were allowed in each testing session, and verbal encouragement was provided during each attempt. The exercises were standardized and continuously monitored by the same experienced rater in an attempt to ensure data quality and determine the load within 3 attempts. The intraclass correlation coefficient for test-retest reliability was $\geq 0.93$ for $10 \mathrm{RM}$ (da Silva et al. 2017) tests, respectively, 
indicating the elimination of the learning curve for the participants. All tests sessions were performed at the same location between 8:00 and 10:00 am.

\section{Resistance exercise session}

On days 1 and 3 of supplementation, both groups underwent a session of RE involving the hack squat and leg press exercises (3 sets at 80\% of 10RM) (Nakagym equipment, São Paulo, Brazil), with 2 min of rest between sets and exercises. A set was completed when reaching 12 repetitions or as many fewer repetitions as possible before reaching volitional fatigue. The cadence of muscle action was $1 \mathrm{~s}$ concentric: $2 \mathrm{~s}$ eccentric according to a metronome. This protocol was designed to maximize the recruitment of quadriceps muscle, and the training stimulus was similar to a conventional RE session for novice individuals (ACSM, 2009). We have chosen to use the 10RM load (as opposed to $1 \mathrm{RM}$ ) due to its easy application in the practical context and safety for novice practitioners. RE session began with general (moderate walking on treadmill for $10 \mathrm{~min}$ ) and specific (12 repetitions in the leg press, with a self-selected load) warm-up exercises for quadriceps muscle. Qualified personnel (e.g., personal trainer and physiologist) supervised each participant individually during the sessions, and the number of repetitions was registered. The RE sessions were performed between 8:00 and 10:00 a.m.

\section{Supplementation}

The groups were supplemented orally with an identical looking and equivalent amount of BCAA (8 g) plus $4 \mathrm{~g}$ maltodextrin (Arboretum ${ }^{\circledR}$, Londrina, PR, Brazil) or $12 \mathrm{~g}$ of placebo-maltodextrin, dissolved into $300 \mathrm{~mL}$ of water. Maltodextrin was added to ensure that the drinks were indistinguishable in taste. The BCAA drink contained a 2:1:1 ratio of leucine ( $4 \mathrm{~g})$, isoleucine ( $2 \mathrm{~g}$ ), and valine ( $2 \mathrm{~g}$ ), and was consumed (identical to placebo group), 15 min prior to RE sessions and functional tests (days 1, 3, 
4 and 5) and at breakfast on day off (day 2). BCAA drink (or placebo) were ingested 15 min prior to RE sessions and functional tests based on previous studies that reported (i) an elevation in plasma BCAA concentrations within 15 min and a peak 30 min after ingestion of a 5.5-g BCAA mixture (Shimomura et al., 2006), (ii) a greater postexercise muscle-protein synthesis when an essential amino acid-carbohydrate mixture was consumed before exercise, rather than after (Tipton et al., 2001), (iii) a decrease in muscle-protein breakdown when BCAA was consumed before exercise (MacLean et al., 1994), and (iv) a attenuation in muscle damage and loss of strength during recovery when a 5.5-g BCCA mixture was consumed $15 \mathrm{~min}$ prior to high-intensity RE (Shimomura et al. 2010). Moreover, we chose to provide $8 \mathrm{~g}$ of BCAA because lower doses (2.5-5.5 g) have been shown to increase the plasma BCAA concentration (Shimomura et al. 2010; Fouré et al. 2016). To ensure the double-blind design, an individual who was not involved in the study was responsible for placing the supplements into bags and labeling the capsules with the subjects’ names according to the randomization list.

\section{Nutrient intake}

Participants completed a 3-day dietary intake record between RE sessions (days 1-3). The macronutrient composition of the diets was calculated using software for nutritional assessment (Avanutri version 3.1.4, Avanutri Equipamentos de Avaliação, Ltda, Rio de Janeiro, Brazil). Participants were instructed to duplicate their food intake for the $24 \mathrm{~h}$ preceding each session and to refrain from any strenuous activity during the experimental period. The participants were also instructed to report any adverse events from the supplements. No discomfort or adverse effects were reported after BCAA ingestion. 


\section{Perceived exertion}

Rating of perceived exertion was measured immediately after each set of leg press exercise in the last RE session (day 3) using the OMNI-RES scale (Robertson et al. 2003). The participants were instructed to report the perceived exertion value by indicating a number on the OMNI-RES scale (0 for "no effort" and 10 for "maximal effort”) that best represented their overall muscular effort (Robertson et al. 2003; Marcora, 2009). The score was the value (0-10) reported in OMNI-RES scale. All participants were familiar with the OMNI-RES scale before starting the study.

\section{Muscle soreness}

Muscle soreness was measured at baseline (day 0), and $30 \mathrm{~min}$. (day 3), 24 h (day 4) and $48 \mathrm{~h}$ (day 5) after last RE session, using a visual analog scale (VAS). The VAS consisted of a 10-cm line whose endpoints were labeled with "no pain" (left) and “unbearable pain” (right) (Bijur et al. 2001). Participants were asked to perform two functional movements (walk up a flight of stairs, and walk down a flight of stairs) and then mark a vertical line at a scale point that best represented their rating of momentary soreness on quadriceps muscle (Rindom et al. 2016). The score was the distance (in centimeters) from the left side of the scale to the point marked (Mattacola et al. 1997). The ICC of the VAS for acute soreness was $\geq 0.97$ (Bijur et al. 2001).

\section{Countermovement jump}

Participants performed a CMJ on a mobile jump mat (Smart Jump, Fusion Sport, Australia) to assess maximal jumping height, and peak power output/body weight ratio (PPO/BW) (Rindom et al. 2016; Waldron et al. 2017). Participants were given three attempts interspaced by 1 min of recovery. The best of the three attempts was used for further analysis. Qualified personnel individually supervised each participant during 
test. The test re-test reliability of this procedure was 1.2\% (Coefficient of variation) (Waldron et al. 2017)

\section{Statistical analysis}

Data are expressed as means \pm standard deviation (SD). The normality and homogeneity for outcome measures were tested using Shapiro-Wilk's and Levene's tests, respectively. The independent variables included the experimental groups (i.e., Placebo and BCAA) and time (i.e., 30 min., 24 h, and 48 h). Dependent variables included number of repetitions, rating of perceived exertion, muscle soreness, jumping height, and PPO/BW. Baseline characteristics and nutritional intake were compared between groups using an unpaired t-test. Two-way (treatment $\mathrm{x}$ time) analysis of variance (ANOVA) with repeated measures was used to evaluate changes over time and between groups for all dependent variables. Violation of sphericity was corrected using the Greenhouse-Geisser method. When significant differences were confirmed with ANOVA, multiple comparisons testing were performed using Bonferroni post hoc correction to identify these differences. The significance level was set at $P \leq 0.05$. Statistical analyses were performed using SPSS Statistics for Windows version 20.0 (IBM Corp., Armonk, N.Y., USA). 


\section{RESULTS}

Baseline characteristics and food intake. The BCAA and Placebo groups had similar $(P>0.05)$ baseline characteristics $($ Table 1$)$. There were no significant $(P>0.05)$ differences between groups in daily nutritional intake during the days of training (Table 2), and both groups had adequate macronutrient intakes according to the recommendations of the American College of Sports Medicine (Thomas et al. 2016).

\section{** Table 1 about here \\ ** Table 2 about here **}

Number of repetitions and perceived exertion. There was no significant time effect $(P>0.05)$ or group $\mathrm{x}$ time interaction $(P>0.05)$ in number of repetitions among the sets (Set 1 to 3) of the leg press exercise during last RE session (Fig. 2A). There was a significant (time, $P<0.05$ ) increase in perceived exertion among the 3 sets of the leg press (Set 1 to 3) exercise during the last RE session (Fig. 2B), with no difference (group x time, $P>0.05$ ) between the groups.

\section{** Figure 2 about here **}

Muscle soreness. There was no significant group $\mathrm{x}$ time interaction $(P>0.05)$, but a significant main effect for time $(P<0.05)$ indicated an increase in muscle soreness during walking up (Fig 3A) and down stairs (Fig. 3 B) from basal (pre-RE session) to 30 min. postexercise, and progressively returned to baseline at 24 and 48 h post RE session in both groups (time, $P<0.05$ ).

\section{** Figure 3 about here **}

Countermovement jump. There was no significant group $\mathrm{x}$ time interaction $(P>0.05)$, but a significant main effect for time $(P<0.05)$ indicated a decrease in jumping height and PPO/BW from basal (pre-RE session) to $30 \mathrm{~min}$. postexercise, and returned to baseline at 24 h post RE session in both groups (time, $P<0.05$ ) (Fig. 4). 


\section{** Figure 4 about here **}

\section{DISCUSSION}

The purpose of this study was to investigate the effects of BCAA supplementation on muscle recovery from $\mathrm{RE}$ in untrained young adults. Considering previous positive findings on muscle anabolism following exercise (Karlsson et al., 2004; Blomstrand et al. 2006; Jackman et al. 2017), we hypothesized that BCAA supplementation would ameliorate the post-exercise negative symptoms (i.e., soreness and loss of muscle function) associated with RE stimulus. Contrary to our hypothesis, we found no evidence indicating that BCAA supplementation could alleviate the alterations of muscle soreness and function (i.e., number of repetitions, perceived exertion, jumping height, and PPO/BW) during time course of recovery from RE.

It has been postulated that the beneficial effects of BCAA are evident when exercise results in a low-to-moderate, but not severe muscle damage (Fouré et al. 2016; Fouré and Bendahan, 2017). For example, Aminiaghdam et al. (2012) and Fouré et al. (2016) found no beneficial effects of BCAA supplementation on markers of recovery (i.e., maximal voluntary isometric contraction, soreness, and concentrations of phosphocreatine [PCr], inorganic phosphate [Pi] and $\mathrm{pH}$ ) in response to an exerciseinduced severe damage (peak CK levels ranged from 660 to $17211 \mathrm{IU} / \mathrm{L}$ ), while other authors (Jackman et al. 2010; Shimomura et al. 2010; Howatson et al. 2012) showed an attenuation of muscle soreness when a low-to-moderate damage was achieved (peak CK levels ranged from 80 to $400 \mathrm{IU} / \mathrm{L})$. However, our results do not support this supposition that the positive effects of BCAA are dependent on the level of muscle damage. Although we did not analyze CK levels in current study, previous findings from our laboratory (da Silva et al. 2017; Andrade et al. 2018) showed peak CK values around 300-350 IU/L using similar RE protocols. Therefore, even with a moderate 
exercise-induced muscle damage, BCAA supplementation failed to alleviate muscle soreness compared to the placebo group, suggesting that the relationship between BCAA supplementation and muscle damage is unwarranted.

It is noteworthy that CK levels may be ambiguous for analyzing the impact of BCAA supplementation on muscle soreness, since a positive effect of BCAA was found on muscle soreness (Jackman et al. 2010; Waldron et al., 2017), even when CK levels were unchanged (Jackman et al. 2010, Shimomura et al. 2010) or higher (Waldron et al., 2017) in relation placebo. Moreover, a large interindividual variability is often observed in CK levels in response to muscle damage (Clarkson and Ebbeling, 1988) and there is no correlation between the increase in CK and loss of muscle strength (Jackman et al. 2010). These findings support the idea that CK levels might not be an appropriate indirect marker of muscle damage (Chrismas et al. 2013), and suggest that complementary markers (e.g., soreness and strength) should be used in the context of muscle recovery. Regardless of the limitations involving CK levels, our results indicate that BCAA supplementation is unable attenuate muscle soreness over the time course of recovery from RE, corroborating recent findings from a well-conducted meta-analysis study (Rahimi et al. 2017).

In addition to soreness, we observed no effect of BCAA supplementation on muscle function between the two RE sessions (i.e., number of repetitions and perceived exertion) and during the recovery period (i.e., jumping height and PPO/BW). Our results are consistent with previous studies that found a small-to-moderate (Waldron et al. 2017) or no beneficial effect of BCAA on muscle function (e.g., strength and power) during recovery from exercise (Jackman et al. 2010; Areces et al. 2014; Fouré et al. 2016; Kephart et al. 2016). On the other hand, some studies have shown that BCAA supplementation may attenuate the decline of muscle strength after exercise (Greer et al. 
2007; Shimomura et al. 2010; Howatson et al. 2012; Gee and Daniel, 2016). The small amount of studies and wide discrepancy of results found in the literature regarding the extent of muscle damage (EMD) and positive effects of BCAA supplementation preclude a more in-depth discussion about our findings. For example, among the 4 studies that found positive effects of BCAA on muscle function, 3 reported low EMD and 1 reported moderate EMD (according to criteria suggested by Fouré and Bendahan, 2017); while among the 5 studies that found a small-to-moderate or no positive effect of BCAA, 2 reported low EMD and 2 high EMD and 1 moderate EMD. A likely explanation for this discrepancy in EMD may be the wide methodological variety (e.g., type of exercise, timing and period of supplementation, and studied sample) among studies. Therefore, this mix of discordant results indicate that relationship between EMD and positive effects of BCAA supplementation on muscle function during exercise recovery seems still unclear. Our results add for this assertion by showing that BCAA supplementation does not improve muscle function during recovery from a moderate EMD induced by RE.

The lack of effect of BCAA supplementation found in our study does not seem to be explained by the supplementation strategy (i.e., duration, frequency, and dosage), since most studies that found positive results used similar supplementation strategies (according to criteria suggested by Fouré and Bendahan, 2017) (duration: short [3 days or less] to long [more than 10 days], frequency: low [less than 2 intakes per day] to high [2 or more intakes per day], and dosage: low [ $<200 \mathrm{mg} / \mathrm{kg} / \mathrm{d}]$ to high [ $\geq 200 \mathrm{mg} / \mathrm{kg} / \mathrm{d}]$ ) (Greer et al. 2007; Shimomura et al. 2010; Howatson et al. 2012; Gee and Daniel, 2016) in comparison with our study and others with negative findings (duration: short [3 days or less] to moderate [between 4 and 10 days], frequency: low [less than 2 intakes per day] to high [2 or more intakes per day], and dosage: low [ $<200 \mathrm{mg} / \mathrm{kg} / \mathrm{d}]$ to high $[\geq$ 
$200 \mathrm{mg} / \mathrm{kg} / \mathrm{d}]$ ) (Waldron et al. 2017; Jackman et al. 2010; Areces et al. 2014; Fouré et al. 2016; Kephart et al. 2016). It is important to note that among studies that found positive effects only one (Howatson et al. 2012) used a longer supplementation period (more than 10 days), while others (Greer et al. 2007; Shimomura et al. 2010; Gee and Daniel, 2016) used a shorter duration (3 days or less). Moreover, in two of these studies (Greer et al. 2007; Shimomura et al. 2010) the duration, frequency and dosage were short (1 day), low (<2x/day) and low ( 5 g), respectively, suggesting that supplementation strategy was not the key factor for positive outcomes. Interestingly, in our study the duration ( 5 days) and dosage ( 8 g) was longer and the frequency (1x/day) similar compared to the studies of Greer et al. (2007) and Shimomura et al. (2010), reinforcing the notion that the interaction between BCAA supplementation and muscle recovery may be associated with other factors not related to the strategy of supplementation. Therefore, in the light of our findings and discrepancy among previous studies it seems premature to consider BCAA supplementation as a potential strategy to improve the muscle recovery from exercise.

It is important to mention that an BCAA dose $<8.0 \mathrm{~g}$ is sufficient to significantly increased plasma BCAA concentrations in humans (Shimomura et al., 2006; Fouré et al. 201). Thus, the lack of a positive effect of BCAA supplementation on muscle recovery from RE does not necessarily indicate that our participants did not experience an increase in muscle BCAA levels. However, we cannot rule out the possibility that daily protein intake ( $1.5 \mathrm{~g} / \mathrm{kg} / \mathrm{d})$ might have been sufficient to maximize muscle recovery in our participants, masking the BCAA effects. The analysis of daily nutritional intake reinforces the internal validity of our study compared to previous studies in this area, since most studies that found positive effects of BCAA on muscle recovery markers did 
not analyze the participants' protein intake, precluding to determine whether the effects of BCAA supplementation were independent of the daily protein intake.

A few limitations of this study must be mentioned. First, we did not analyze blood amino acids levels to confirm the absorption of BCCA. However, previous studies using lower doses have shown an increase in plasma BCAA concentration (Shimomura et al. 2010; Fouré et al. 2016). Second, we did not collect muscle biopsies for analysis of direct markers of muscle regeneration (e.g., IGF-I, mTOR, and p70S6 k); however, we analyzed the soreness levels and muscle power as tissue and functional markers of muscle recovery after exercise (Rindom et al. 2016). Finally, we decided to analyze the markers of muscle recovery only after the second exercise session to generate a cumulative effect of the mechanical/physiological stress between two sessions and thus mimic a practical resistance training routine; however, we cannot exclude the possibility that the effects of the first session may have somewhat influenced the response of the second session and thus the recovery process between groups.

In conclusion, our data indicate that BCAA supplementation does not improve muscle soreness and function during recovery from RE in untrained young adults. These results bring into question the ergogenic potential of BCAA for improving muscle recovery in healthy individuals, and thereby suggest that other mechanisms may be involved in the muscle recovery in response to BCAA supplementation. Further studies are required to assess the effects of BCAA supplementation on muscle recovery in other populations (e.g., elderly and/or women), with different training status (e.g., recreational practitioners and/or athletes) and training protocols (e.g., whole-body muscle groups) to better understand the underlying mechanism(s) of this supplement before making any recommendation about its utilization as an ergogenic aid for recovery. 


\section{COMPLIANCE WITH ETHICAL STANDARDS}

Acknowledgement: We address special thanks to all the participants for their engagement in this study.

Funding: This study was funding by North University of Paraná (UNOPAR) (grant number: 02.2016).

Conflict of interest: The authors declare that they have no conflict of interest.

Informed consent: This research involved human participants, who were carefully informed of the procedures, risks, and benefits of the investigation and signed an informed consent document approved by the research ethics committee of the University. 


\section{REFERENCES}

1. American Collge of Sports Medicine position stand (ACSM) (2009) Progression models in resistance training for healthy adults. Med Sci Sports Exerc 41(3):687708.

2. Aminiaghdam S, Baturak K, Panahi PM, Khaidan Hatami (2012) The effects of BCAA supplementation on muscle damage following a lower-body resistance exercise bout in soccer players. Football Science 9:62-69.

3. Andrade WB, Jacinto JL, da Silva DK, Roveratti MC, Estoche JM, Oliveira DB, et al. (2018) l-Arginine supplementation does not improve muscle function during recovery from resistance exercise. Appl Physiol Nutr Metab 43(9):928-936.

4. Areces F, Salinero JJ, Abian-Vicen J, Gonzalez-Millan C, Gallo-Salazar C, RuizVicente D. et al. (2014) A 7-day oral supplementation with branched-chain amino acids was ineffective to prevent muscle damage during a marathon. Amino Acids 46(5):1169-76.

5. Bijur PE, Silver W, Gallagher EJ (2001) Reliability of the visual analog scale for measurement of acute pain. Acad Emerg Med 8(12):1153-1157.

6. Blomstrand E, Eliasson J, Karlsson H, Kohnke R (2006) Branched-chain amino acids activate key enzymes in protein synthesis after physical exercise. J Nutr 136(1):269S-73S.

7. Borgenvik M, Apró W, Blomstrand, E (2012) Intake of branched-chain amino acids influences the levels of MAFbx mRNA and MuRF-1 total protein in resting and exercising human muscle. Am J Physiol Endocrinol Metab 302(5):E510-21.

8. Chrismas BCR, Taylor L, Carrol S, Smith A, Pemberton P, Seigler JC et al. (2009) Reproducibility of creatine kinase: how useful is this measurement tool? 11th ISEI Symposioum, Newcastle, Australia, 9-12 Sept. 2013. Exercise Immunology: 


\section{Prescriptions for Health.}

9. Clarkson PM, Ebbeling C (1988) Investigation of serum creatine kinase variability after muscle-damaging exercise. Clin Sci (Lond) 75(3):257-61.

10. da Silva DK, Jacinto JL, de Andrade WB, Roveratti MC, Estoche JM, Balvedi MCW, et al. (2017) Citrulline malate does not improve muscle recovery after resistance exercise in untrained young adult men. Nutrients 9(10):E1132.

11. Fouré A, Bendahan D (2017) Is branched-chain amino acids supplementation an efficient nutritional strategy to alleviate skeletal muscle damage? A systematic review. Nutrients 9(10):E1047.

12. Fouré A, Nosaka K, Gastaldi M, Mattei JP, Boudinet H, Guye M, et al. (2016) Effects of branched-chain amino acids supplementation on both plasma amino acids concentration and muscle energetics changes resulting from muscle damage: A randomized placebo controlled trial. Clin Nutr 35(1):83-94.

13. Gee T, Deniel S (2016) Branched-chain amino acid supplementation attenuates a decrease in power-producing ability following acute strength training. J Sports.Med Phys Fitness 56(12):1511-1517.

14. Greer BK, Woodard JL, White JP, Arguello EM, Haymes EM (2007) Branchedchain amino acid supplementation and indicators of muscle damage after endurance exercise. Int J Sport Nutr Exerc Metab 17(6):595-607.

15. Howatson G, Hoad M, Goodall S, Tallent J, Bell PG, French DN (2012) Exercise induced muscle damage is reduced in resistance-trained males by branched chain amino acids: a randomized, double-blind, placebo controlled study. J Int Soc Sports Nutr 9:20.

16. Jackman SR, Witard OC, Jeukendrup AE, Tipton KD (2010) Branched-chain amino acid ingestion can ameliorate soreness from eccentric exercise. Med Sci 
Sports Exerc 42(5):962-70.

17. Karlsson H, Nilsson P, Nilsson J, Chibalin A, Zierath J, Blomstrand E (2004) Branched-chain amino acids increase p70S6k phosphorylation in human skeletal muscle after resistance exercise. Am J Physiol Endocrinol Metab 287(1):E1-7.

18. Kephart W, Wachs T, Mac Thompson R, Brooks Mobley C, Fox C, McDonald JR. et al. (2016) Ten weeks of branched-chain amino acid supplementation improves select performance and immunological variables in trained cyclists. Amino Acids 48(3):779-789.

19. Kim DH, KimS-H, Jeong WS, Lee HY (2013) Effect of BCAA intake during endurance exercises on fatigue substances, muscle damage substances, and energy metabolism substances. J Exerc Nutr Biochem 17(4):169-80.

20. Knechtle B, Mrazek C, Wirth A, Knechtle P, Rust CA, Senn O, et al. (2012) Branched chain amino acid supplementation during a 100-km ultra-marathon-a randomized controlled trial. J Nutr Sci Vitaminol (Tokyo) 58(1):36-44.

21. Layman DK, Baum JI (2004) Dietary protein impact on glycemic control during weight loss. J Nutr 134(4):968S-73S.

22. MacLean DA, Graham TE, Saltin B (1994) Branched chain amino acids augment ammonia metabolism while attenuating protein breakdown during exercise. Am J Physiol 267(6 Pt 1):E1010-22.

23. Marcora S (2009) Perception of effort during exercise is independent of afferent feedback from skeletal muscles, heart, and lungs. J Appl Physiol 106(6):2060-2062.

24. Mattacola CG, Perrin DH, Gansneder BM, Allen JD, Mickey CA (1997) A Comparison of Visual Analog and Graphic Rating Scales for Assessing Pain Following Delayed Onset Muscle Soreness. J Sport Rehabil 6(1):3 8-46.

25. Nosaka K, Sacco P, Mawatari K (2006) Effects of amino acid supplementation on 
muscle soreness and damage. Int J Sport Nutr Exerc Metab 16:620-635.

26. Pasiakos SM, Lieberman HR, McLellan TM (2014) Effects of protein supplements on muscle damage, soreness and recovery of muscle function and physical performance: a systematic review. Sports Med 44(5):655-70.

27. Ra SG, Miyazaki T, Ishikura K, Nagayama H, Komine S, Nakata Y, et al. (2013) Combined effect of branched-chain amino acids and taurine supplementation on delayed onset muscle soreness and muscle damage in high-intensity eccentric exercise. J Int Soc Sports Nutr 10(1):51.

28. Rahimi MH, Shab-Bidar S, Mollahosseini M, Djafarian K (2017) Branchedchain amino acid supplementation and exercise-induced muscle damage in exercise recovery: A meta-analysis of randomized clinical trials. Nutrition 42:30-36.

29. Riazi R, Wykes LJ, Ball RO, Pencharz PB (2003) The total branched-chain amino acid requirement in young healthy adult men determined by indicator amino acid oxidation by use of L-[1-13C]phenylalanine. J Nutr 133(5):1383-9.

30. Rindom E, Nielsen MH, Kececi K, Jensen ME, Vissing K, Farup J (2016) Effect of protein quality on recovery after intense resistance training. Eur $\quad \mathrm{J}$ Appl Physiol 116(11-12):2225-2236.

31. Robertson RJ, Goss FL, Rutkowski J, Lenz B, Dixon C, Timmer J, et al. (2003) Concurrent validation of the OMNI perceived exertion scale for resistance exercise. Med Sci Sports Exerc 35(2): 333-41.

32. Sharp CP, Pearson DR (2010) Amino acid supplements and recovery from high intensity resistance training. J Strength Cond Res 24(4):1125-30.

33. Shimomura Y, Inaguma A, Watanabe S, Yamamoto Y, Muramatsu Y, Bajotto G, et al. (2010) Branched-chain amino acid supplementation before squat exercise and delayed-onset muscle soreness. Int J Sport Nutr Exerc Metab 20(3):236-244. 
34. Shimomura Y, Yamamoto Y, Bajotto G, Sato J, Murakami T, ShimomuraN, et al. (2006) Nutraceutical effects of branched-chain amino acids on skeletal muscle. J Nutr 136(2):529S-532S.

35. Thomas DT, Erdman KA, Burke LM (2016) American College of Sports Medicine Joint Position Statement. Nutrition and athletic performance. Med Sci Sports Exerc 48(3): 543-568.

36. Tipton KD, Rasmussen BB, Miller SL, Wolf SE, Owens-Stovall SK, Petrini BE, et al. (2001) Timing of amino acid-carbohydrate ingestion alters anabolic response of muscle to resistance exercise. Am J Physiol Endocrinol Metab 281(2):E197-E206.

37. Waldron M, Whelan K, Jeffries O, Burt, D, Howe L, Patterson SD (2017) The effects of acute branched-chain amino acid supplementation on recovery from a single bout of hypertrophy exercise in resistance-trained athletes. Appl Physiol Nutr Metab 42(6):630-636.

38. Wolfe R (2017) Branched-chain amino acids and muscle protein synthesis in humans: myth or reality? J Int Soc Sports Nutr 14:30. 


\section{FIGURE LEGENDS}

Figure. 1. Experimental design.

Figure. 2. Number of repetitions (A) and perceived exertion (B) over 3 sets of leg press exercise in the last training session in the BCAA $(N=12)$ and Placebo $(N=12)$ groups. Data are means \pm SD. Difference letters indicate significant difference over time at $P<$ 0.05 .

Figure. 3. Muscle soreness during walking up (A) and walking down (B) a flight of stairs at baseline (pre-exercise) and during the recovery period (30 min., $24 \mathrm{~h}$, and $48 \mathrm{~h}$ postexercise $)$ in the BCAA $(N=12)$ and Placebo $(N=12)$ groups. Data are means \pm SD. Difference letters indicate different from each other and compared to baseline at $P<$ 0.05 .

Figure. 4. Jumping weight (A) and peak power output/body weight (PPO/BW) (B) at baseline (pre-exercise) and during the recovery period (30 min., $24 \mathrm{~h}$, and $48 \mathrm{~h}$ postexercise $)$ in the BCAA $(N=12)$ and Placebo $(N=12)$ groups. Data are means \pm SD. ${ }^{\text {a }} \mathrm{P}<0.05$ compared to basal, $24 \mathrm{~h}$, and $48 \mathrm{~h}$. 